\title{
Aqueous-Mediated Synthesis and Characterization of Gelatin Methacryloyl for Biomedical Applications
}

\author{
Saravanan Ramesh ${ }^{1}$ (D), Murugan Ramalingam 2,* (D) \\ 1 School of Bio Sciences and Technology, Vellore Institute of Technology, Vellore 632014, India \\ 2 Biomaterials and Organ Engineering Group, Centre for Biomaterials, Cellular and Molecular Theranostics, School of \\ Mechanical Engineering, Vellore Institute of Technology, Vellore 632014, India \\ * Correspondence: rmurug2000@gmail.com (M.R.);
}

Scopus Author ID 7007046854

Received: 29.06.2021; Revised: 15.09.2021; Accepted: 19.09.2021; Published: 8.11.2021

\begin{abstract}
In recent years, gelatin methacryloyl (GelMA)-based hydrogels have been widely used in many biomedical applications, particularly tissue engineering, due to their biocompatibility and tunable physicochemical, mechanical, and other functional properties. Phosphate buffer saline (PBS) or carbonate-bicarbonate buffer are frequently used to make GelMA. In this article, the authors provide an elegant aqueous-mediated synthesis and characterization of GelMA. The experimental conditions were optimized and extensively discussed. The results of proton nuclear magnetic resonance $\left({ }^{1} \mathrm{H} N M R\right)$ and Fourier transform infrared spectroscopy (FTIR) confirmed the formation of GelMA with characteristic absorption peaks and chemical functional groups. The morphological analysis done with scanning electron microscopy (SEM) showed the porous nature of the GelMA with an average pore size of 80 microns. It was also found that the gelation properties of aqueous-mediated GelMA hydrogel were highly tunable by varying the percentage of gel concentration, which yields a wide range of tunable physicochemical properties. The preparation of this kind of aqueous-mediated GelMA is cost-effective compared to their counterparts and easy to prepare in laboratory settings with high yield under optimal conditions.
\end{abstract}

Keywords: gelatin methacryloyl; photopolymerization; gelation; hydrogel; biomedical applications.

(C) 2021 by the authors. This article is an open-access article distributed under the terms and conditions of the Creative Commons Attribution (CC BY) license (https://creativecommons.org/licenses/by/4.0/).

\section{Introduction}

The last few decades have witnessed considerable interest in and utilization of hydrogels in various biomedical applications due to their adjustable physicochemical and mechanical properties. Hydrogels are a three-dimensional (3D) network of hydrophilic polymers, which can absorb a large amount of water and maintain the network structure in a swollen state. However, the choice of polymeric material is the key to properly employing hydrogels for their desired application. For example, choosing a highly biocompatible polymer is essential for preparing hydrogel suitable for biomedical applications, particularly tissue engineering.

The ultimate aim of tissue engineering is to devise a functional tissue model for the regeneration and replacement of damaged tissues or organs [1]. In order to accomplish this, it is necessary to fabricate a scaffold using biomaterials and entrap cells within the scaffolding structure, such that the model is capable of mimicking its corresponding in vivo tissue or organ architectures. As a result, the success of the tissue-engineered model depends on choosing the suitable biomaterial and potent cell source to mimic the physical, chemical, and mechanical 
properties of the in vivo native environment [2,3]. The developed biomaterial should support the growth and viability of the biological constituents via its scaffolding nature, matrix stiffness, matrix porosity, and most of its non-toxic, biodegradable potential [4,5]. Hydrogels are being used as a versatile scaffolding system, supporting the 3D architecture and exhibiting high tunability ranges for various applications such as drug delivery and tissue engineering $[6,7]$. Numerous derivatives of hydrogels that have been successfully reported are mainly obtained from the modified form of natural polymer using synthetic compounds [8,9].

Collagen is a major molecule responsible for cellular attachment in vivo [10]. Collagen blended hydrogels have been developed for tissue engineering approaches, but their application remains limited due to poor mechanical properties [11]. Hyaluronic acid and poly(ethylene glycol) (PEG) have exhibited high mechanical strength and long-term viability of encapsulated cells [12] but are limited by their cell binding ability [13]. Shortage of these cell-responsive elements mainly confined the capacity of cell elongation, migration, proliferation, and differentiation into complex microstructure [14-16] and led to the development of synthetic biomaterials.

Gelatin is a well-known naturally derived polymer generated from the hydrolysis of collagen under high acid or alkali reaction. Gelatin has numerous advantages such as excellent biocompatibility, solubility, degradability, and is readily available. Gelatin-based scaffolds exhibit low antigenic property [17] and possess Arg-Gly-Asp (RGD) peptide sequences favoring cell attachment and rendering scaffold remodeling by matrix metalloproteinase (MMP) enzyme [18]. However, gelatin hydrogels were reported to have a rapid degradation profile and low mechanical strength [19] thus, limiting their biomedical applications. Gelatinbased hydrogel functionalized with methacrylic anhydride was first developed by Van Den Bulcke and the group [20]. Methacrylation is often used to regulate the degradation rate and increase the mechanical property of gelatin [21], thereby rendering the GelMA hydrogel as an ideal biomaterial for various biomedical applications, including tissue engineering, drug delivery, and regenerative medicine [17,22,23].

The GelMA was initially synthesized through the chemical modification of gelatin with methacrylic anhydride under the reaction system of PBS with optimized $\mathrm{pH}$ conditions [24,25]. The recent studies indicated that carbonate-bicarbonate buffer employed for GelMA synthesis was superior to PBS in the context of rendering the deprotonation of free amino groups and buffering capability. However, it is not a cost-effective method when compared to the aqueousmediated method [26,27].

Therefore, the aim of the present study was to introduce a cost-effective facile method to synthesize GelMA with an aqueous medium. The experimental conditions were discussed in detail, the synthesized GelMA was characterized for their physicochemical properties by various analytical techniques, and the results were discussed in detail.

\section{Materials and Methods}

\subsection{Materials.}

Gelatin (Type A, 300 bloom from porcine skin) was purchased from MP Biomedicals (USA). Methacrylic anhydride, Dulbecco's phosphate-buffered saline (DPBS), and 2hydroxy-1-(4-(hydroxyethoxy) phenyl)-2-methyl-1-propanone (Irgacure 2959) were purchased from Sigma-Aldrich (USA). 


\subsection{Synthesis of GelMA.}

Gelatin $10 \%$ (w/v) was added into deionized water, $\mathrm{pH}$ adjusted to 8 , and warmed at $60^{\circ} \mathrm{C}$ until complete dissolution. After $15 \mathrm{~min}$, methacrylic anhydride $20 \%$ (w/v) was added into gelatin at the rate of $0.5 \mathrm{~mL} / \mathrm{min}$. This solution was kept under stirring at $50^{\circ} \mathrm{C}$ to allow synthesis reaction for 2 hours. To remove unreacted products, the mixture was subjected to dialysis by using a dialysis tube $\left(12-14 \mathrm{kDa}\right.$ cutoff) for 3 days at $37^{\circ} \mathrm{C}$. Then, the solution was freeze-dried at $-80^{\circ} \mathrm{C}$ overnight and lyophilized for 3 days to generate GelMA porous milky white spongy foam preserved in the freezer at $-80^{\circ} \mathrm{C}$ until further use.

\subsection{Preparation of GelMA hydrogel.}

For hydrogel preparation, lyophilized GelMA was added into DPBS containing $0.5 \%$ (w/v) of Irgacure 2959 and allowed to dissolve at $80^{\circ} \mathrm{C}$. Hydrogels were prepared at three different concentrations of GelMA (5, 10, and 15\%). Once completely dissolved, GelMA prepolymer solution with photoinitiator was pipette into a glass mold separated by 2-mm spacers. Then, the prepolymer solution was placed under $8 \mathrm{~mW} / \mathrm{cm} 2 \mathrm{UV}$ light intensity at $365 \mathrm{~nm}$ wavelength for a specified time [28].

\section{4. ${ }^{1} \mathrm{H} N M R$ analysis.}

To determine the structure of the GelMA molecules, chemical shift values and degree of methacrylation were evaluated by employing ${ }^{1} \mathrm{H}$ NMR [29]. The NMR spectra for the GelMA sample were obtained at $35^{\circ} \mathrm{C}$ in deuterium oxide $\left(\mathrm{D}_{2} \mathrm{O}\right)$ at a frequency of $500 \mathrm{MHz}$ using an NMR spectrometer.

\subsection{Thermal analysis.}

Thermogravimetry analysis (TGA) and differential scanning calorimetric analysis (DSC) was performed to determine the thermal properties of the samples [30]. TGA thermogram of GelMA was recorded in the temperature range of room temperature to $600^{\circ} \mathrm{C}$ at a level of $20^{\circ} \mathrm{C} / \mathrm{min}$ increase under a nitrogen atmosphere. The DSC study was conducted in a differential scanning calorimeter mode with a reaction temperature of $20^{\circ} \mathrm{C} / \mathrm{min}$ up to $600^{\circ} \mathrm{C}$ under a nitrogen atmosphere.

\subsection{FTIR analysis.}

FTIR analysis was carried out using the $\mathrm{KBr}$ (potassium bromide) pellet method using FTIR spectrophotometer (NicoletTM iSTM 50 FTIR Spectrometer). Gelatin type A, methacrylic anhydride, and GelMA in lyophilized form were separately analyzed. Each sample was finely grounded with $\mathrm{KBr}$ and made into pellet form. FTIR spectra were recorded in the range of 4000 to $400 \mathrm{~cm}^{-1}$ averaging 32 scans per sample [31].

\subsection{Morphological analysis.}

The SEM was used to study the morphology and pore size of the GelMA. The GelMA hydrogel was lyophilized overnight using a freeze dryer before use. The lyophilized samples were mounted on aluminum stumps coated with gold in a sputter coater for 120s. The SEM was performed at $20 \mathrm{kV}$ to analyze the morphology and porous microstructural analysis. The SEM image analysis was carried out by using Image J software [32]. 
2.8. Gelation study.

The gelation study determines the formation of the hydrogel at different concentrations of GelMA as a function of time. To monitor the gelation of GelMA, different GelMA concentrations $(5,10$, and $15 \%)$ were mixed with $0.5 \%$ Irgacure 2959 . The prepolymer solutions with photoinitiators were exposed to UV at $365 \mathrm{~nm}$ at a precise time. Each concentration consists of 3 samples in a group. The gelation time was calculated using a stopwatch. The different concentrations of GelMA hydrogel timing were measured and plotted as a graph.

\subsection{Swelling study.}

To analyze the swelling ratio of GelMA, the gels were formed at different concentrations as described above. Immediately following the hydrogel formation, the samples were immersed in DPBS at $37^{\circ} \mathrm{C}$ for $48 \mathrm{~h}$. Samples were extracted from DPBS, and the swollen weight (Ws) of hydrogel was calculated. Then the samples were freeze-dried, and the dry weight of hydrogel $(\mathrm{Wd})$ was calculated. The swelling ratio (SR) was obtained with this formula [33].

$$
\mathrm{SR} \%=(\mathrm{Ws}-\mathrm{Wd}) / \mathrm{Wd} \times 100
$$

\subsection{Degradation study.}

To determine the degradation profile of GelMA, hydrogels with different concentrations were dipped into DPBS with $2.5 \mathrm{U} / \mathrm{mL}$ of collagenase II at $37^{\circ} \mathrm{C}$ for different time intervals $3,6,12,24,36$, and 48h. Then, the undigested hydrogels were cleaned with DPBS, freeze-dried, and their weigh dry mass was evaluated. The degradation rate was obtained by the dry mass of remaining undigested hydrogels divided by the mass of untreated hydrogels [34].

\section{Results and Discussion}

\section{1. ${ }^{1} H$ NMR Analysis.}

GelMA was prepared with $20 \%$ methacrylic anhydride (Figure 1). To obtain the chemically crosslinked GelMA, a carbon double bond was combined by reacting the amine group $\left(\mathrm{NH}_{2}\right)$ of gelatin and carboxyl group $(\mathrm{COOH})$ of methacrylic anhydride. In the ${ }^{1} \mathrm{H} \mathrm{NMR}$ spectra of GelMA, the peaks are interpreted as follows: peaks at 1.14, 1.33, 1.59, and $1.72 \mathrm{ppm}$ are correlated to the methyl residues of the amino acids such as threonine, alanine, lysine, and arginine, respectively; whereas, the appearance of peaks at 2.73, 2.90, 3.15, and 3.55 ppm are correlated to methylene residues of the amino acids such as aspartic acid, lysine, arginine, and proline respectively (Figure 2), and it is also corroborated well with the repaired data [35]. The formation of spectra at 5.3 and $5.6 \mathrm{ppm}$ was attributed to methacrylate bond protons $\left[\mathrm{H}_{2} \mathrm{C}=\mathrm{C}\right.$ $\left.\left(\mathrm{CH}_{3}\right)\right]$ in the ${ }^{1} \mathrm{H}$ NMR study of GelMA. Thus, we observed that methacrylic anhydride was successfully incorporated into the gelatin molecule. The formation of peaks at 1.14 and 2.90 ppm are due to the methacrylic anhydride reaction and suggest that $\mathrm{COOH}$ of methacrylic anhydride primarily interacts with $\mathrm{NH}_{2}$ of gelatin. ${ }^{1} \mathrm{H}$ NMR result of aqueous mediated GelMA correlated with PBS-derived GelMA [36]. 


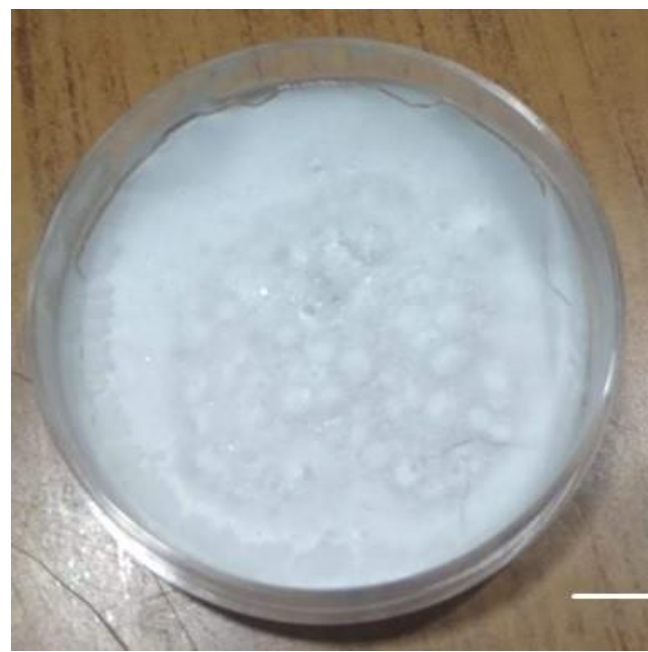

Figure 1. The lyophilized form of GelMA synthesized using $20 \%$ methacrylic anhydride. The scale bar is $3 \mathrm{~cm}$.

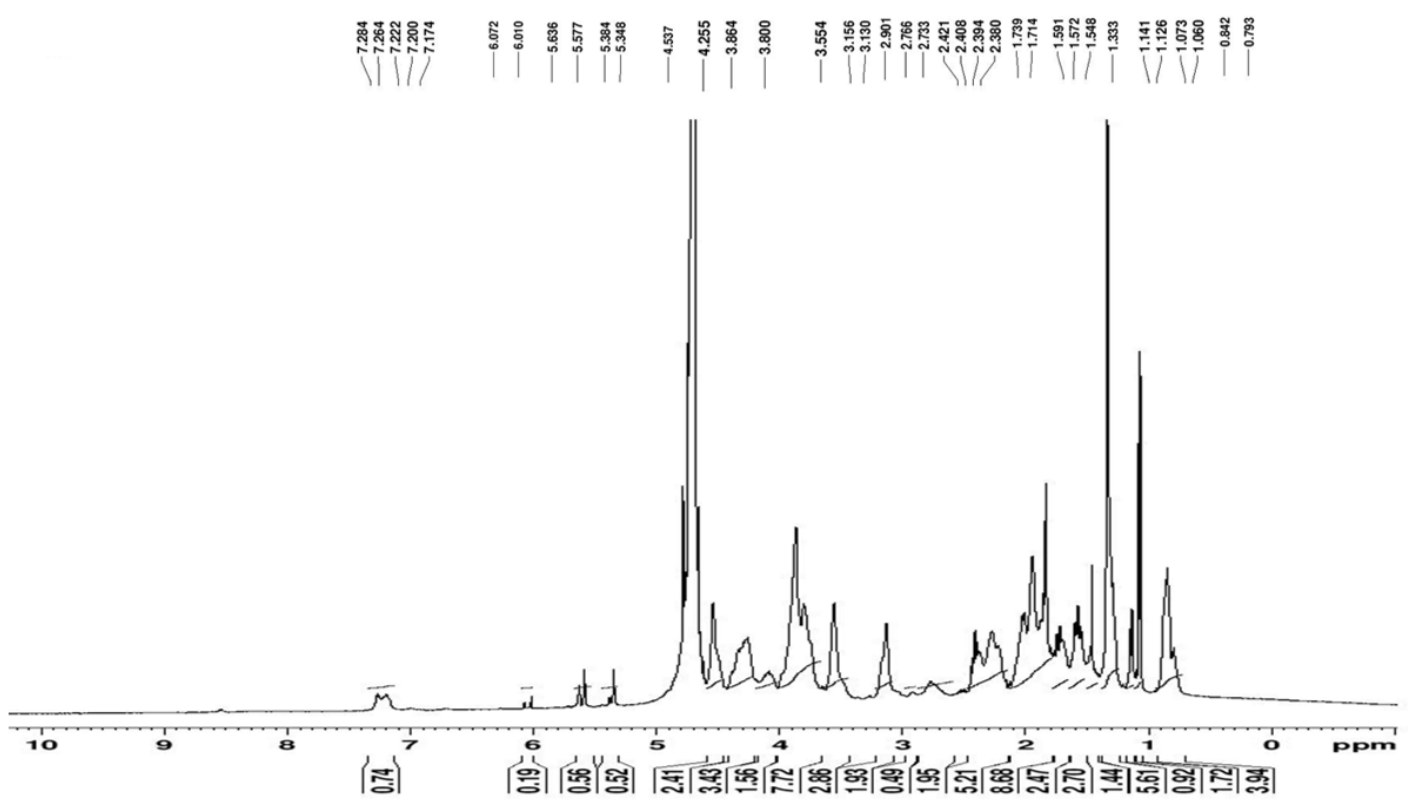

Figure 2. NMR spectrum of GelMA.

\subsection{Thermal analysis.}

The thermal analysis of GelMA was carried out by TGA and DSC. TGA graph reveals that the mass ratio gradually decreased while increasing the temperature (Figure 3A). TGA and DSC thermogram analysis have been conducted simultaneously with a reaction temperature of $20^{\circ} \mathrm{C} / \mathrm{min}$ up to $600^{\circ} \mathrm{C}$ under a nitrogen atmosphere. Derivative weight loss identified in TGA indicates the elimination of physisorbed water from $20-200^{\circ} \mathrm{C}$, the decomposition of organic components (methacrylic anhydride) from $200-450^{\circ} \mathrm{C}$, and combustion of the residual organic matrix from $450-600^{\circ} \mathrm{C}$. TGA graph reveals that the mass ratio of GelMA gradually decreased while increasing the temperature. As shown in the literature, the DSC endothermic peak observed at approximately $75^{\circ} \mathrm{C}$ and $159^{\circ} \mathrm{C}$ is attributed to the denaturation of gelatin and methacrylic anhydride, respectively [37]. In DSC (Figure 3B), the uncrosslinked gelatin Tg value is $81^{\circ} \mathrm{C}$. Therefore, because of the crosslinking reaction, the Tg value of the crosslinked gelatin changed from 81 to $318^{\circ} \mathrm{C}$. Therefore, chemically crosslinked gelatin exhibited a higher $\mathrm{Tg}$ value than observed in uncrosslinked gelatin due to the peptide bonds and crosslinking of methacrylic anhydride functional groups with lysine residue gelatin. 
A

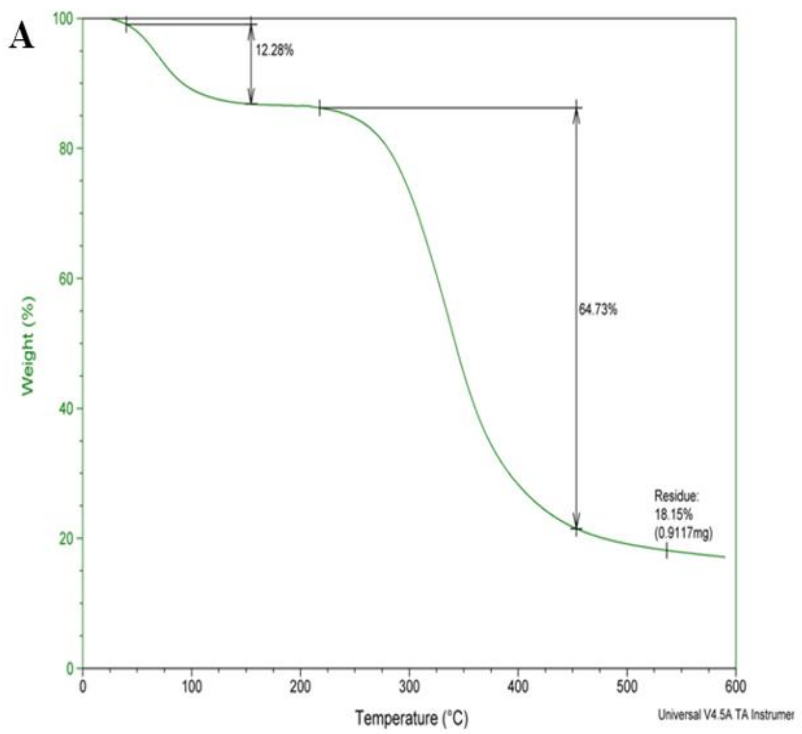

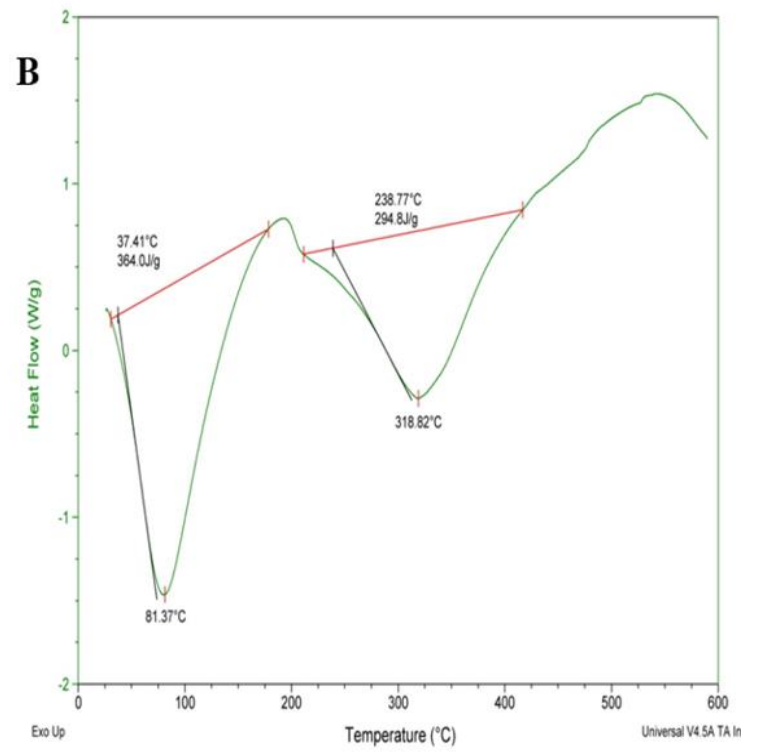

Figure 3. Thermal analysis of GelMA. (A) TGA thermogram and (B) DSC thermogram.

\subsection{FTIR analysis.}

The FTIR spectra of gelatin type A, methacrylic anhydride, and GelMA are shown in Figure 4. The FTIR spectrum of GelMA has the characteristic chemical functional groups and the methacrylic functional groups grafted onto the lysine residue of gelatin [37]. The peak at $3424 \mathrm{~cm}^{-1}$ in GelMA is attributed to the presence of a functional amine group with $\mathrm{N}-\mathrm{H}$ stretching. The peaks at $2924 \mathrm{~cm}^{-1}$ (C-H stretching of alkenes) and $2853 \mathrm{~cm}^{-1}$ (C-H stretching of alkanes) in GelMA denote the presence of gelatin and methacrylic anhydride functional groups between the range from 2500 to $3500 \mathrm{~cm}^{-1}$. The formation of new peaks observed at 2360 and $2342 \mathrm{~cm}^{-1}$ found in GelMA is attributed to $\mathrm{CO}_{2}$ formation in asymmetric stretching mode.

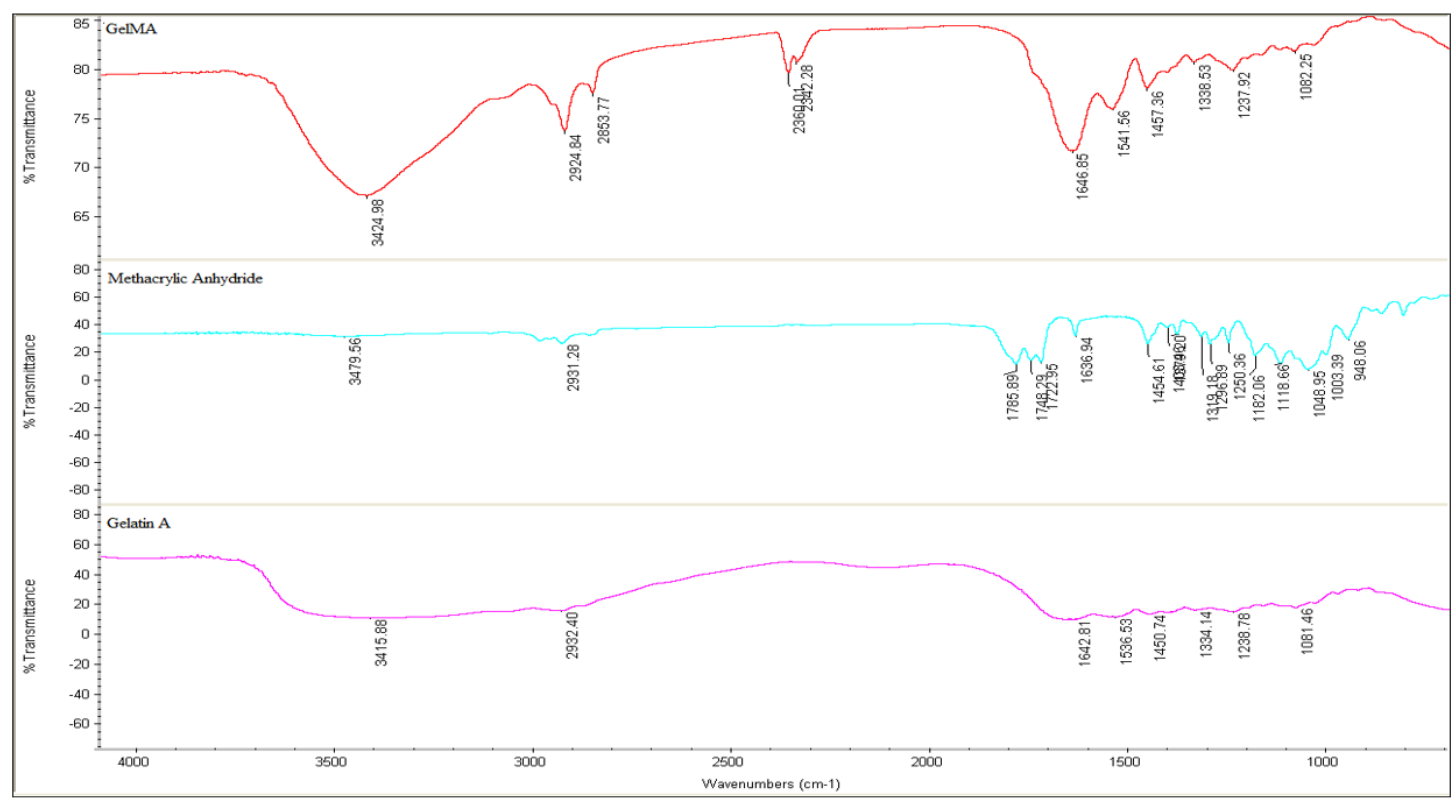

Figure 4. FTIR spectra of gelatin type A, methacrylic anhydride, and GelMA.

The strong band between $1690-1760 \mathrm{~cm}^{-1}$ in methacrylic anhydride indicates the carbonyl $(\mathrm{C}=\mathrm{O})$ group such as carboxylic acid, but the peaks at $1785 \mathrm{~cm}^{-1}$ and $1748 \mathrm{~cm}^{-1}$ disappeared in GelMA due to the release of methacrylic acid in GelMA preparation. The peak at $1646 \mathrm{~cm}^{-1}$ in GelMA denotes the presence of amide $\mathrm{I}$ band, which represents $\mathrm{C}=\mathrm{O}$ stretching. 
The disappearance of the unsaturated group of methacrylic anhydride at $1636 \mathrm{~cm}^{-1}$ in the GelMA spectrum and the formation of amide linkage characterized by the $1646 \mathrm{~cm}^{-1}$ peak determines the crosslinking of gelatin and methacrylic anhydride. The spectrum of GelMA shows the peaks at $1541 \mathrm{~cm}^{-1}$ for $\mathrm{N}-\mathrm{H}$ deformation of amide II and $1237 \mathrm{~cm}^{-1}$ for $\mathrm{N}-\mathrm{H}$ deformation of amide III bands. Also, it has been reported that the FTIR spectrum of PBSderived GelMA indicates amide bonds due to the chemical modification of gelatin with methacrylic anhydride [38].

\subsection{Morphological analysis.}

The porous microstructure is an important criterion for cell invasion and tissue formation in 3D [39,40]. The pore size of GelMA hydrogel is affected by the degree of substitution. Figure 5 shows the porous morphological structure of the GelMA hydrogel. The average pore size was found to be 80 microns. A highly porous structure is important for oxygen diffusion, mass transfer, supporting cell growth, bringing cell nutrients, and removing waste from the matrix [41-43].

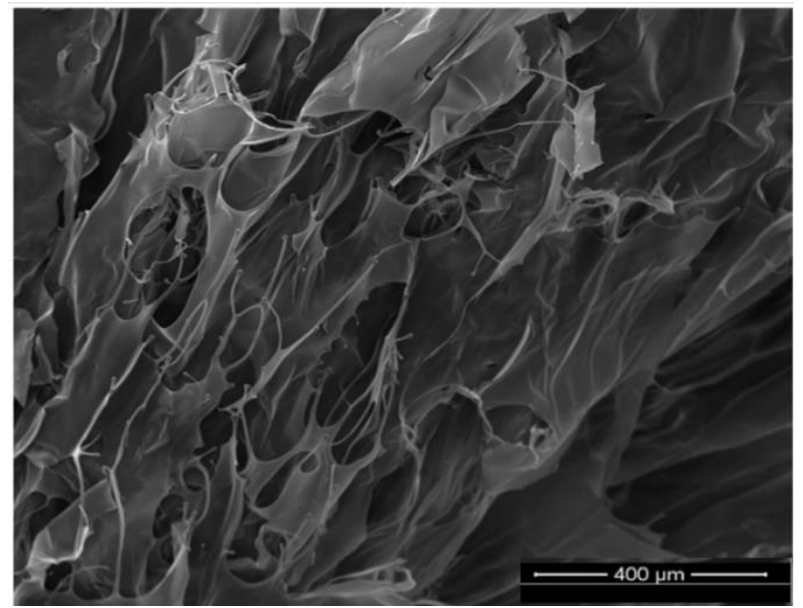

Figure 5. The SEM image represents the porous morphology of synthesized GelMA hydrogel.
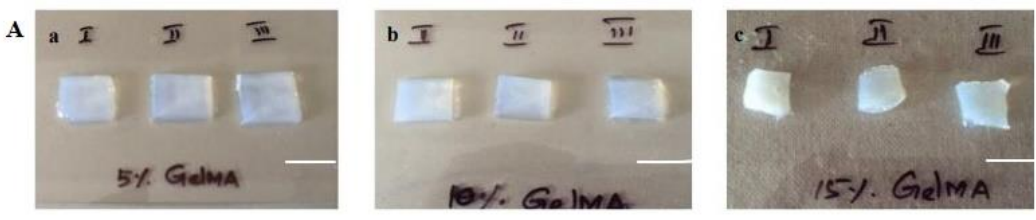

B

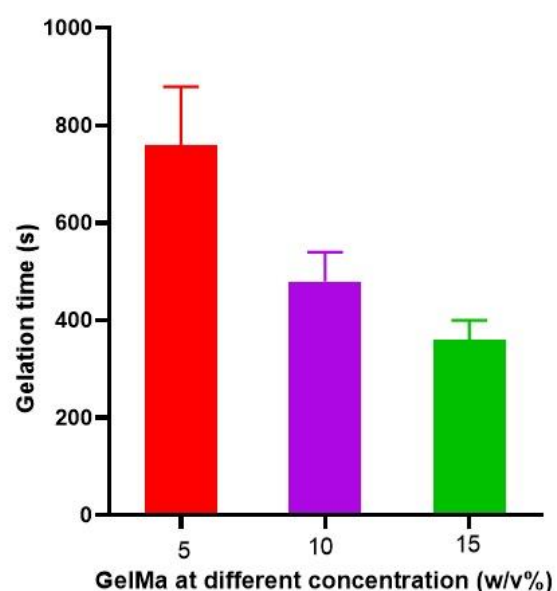

Figure 6. (A) The photographic images of GelMA hydrogels prepared at three different concentrations a) 5\% GelMA, b) $10 \%$ GelMA, and c) $15 \%$ GelMA. Scale bar is $1 \mathrm{~cm}$. (B) The gelation time of GelMA hydrogels. 


\subsection{Gelation study.}

Three different concentrations $(5,10$, and $15 \%)$ of GelMA hydrogels were prepared to analyze the gelation property. As shown in (Figure 6A) the hydrogels were well-formed with a regular shape, and their milky-white color was more profound with the increase of GelMA concentration. The gelation time was carefully determined, determining the period needed to obtain a fully gelled material from the volume of prepolymer solution with a photoinitiator placed under UV exposure. The results showed that the gelation time was inversely proportional to the polymer concentrations. Thus, the results (Figure 6B) show that the increase in polymer concentration induced a decrease in gelation time.

\subsection{Swelling study.}

Figure 7 shows the swelling behavior of the GelMA hydrogel. The data show that the increase in hydrogel concentration decreased the swelling ratio. The variability of the swelling ratio represents the ability and tendency of materials to retain water inside the polymer matrix. Hydration has a substantial effect on physical properties, surface mobility, and surface properties. The swelling ratio of GelMA tends to depend on the gel concentration, chemical reaction of the polymer with solvent, and diameter of pore present in polymer structure [44].

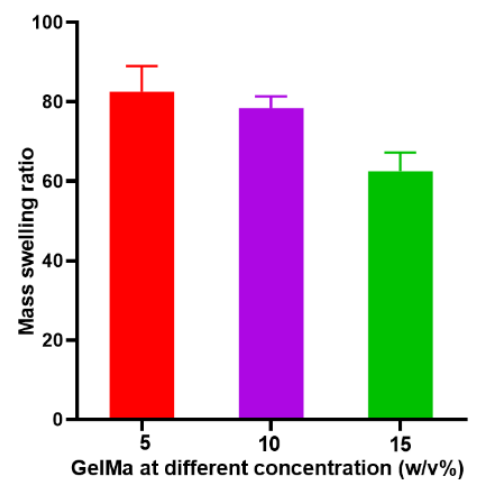

Figure 7. The swelling ratio of GelMA at different concentrations.

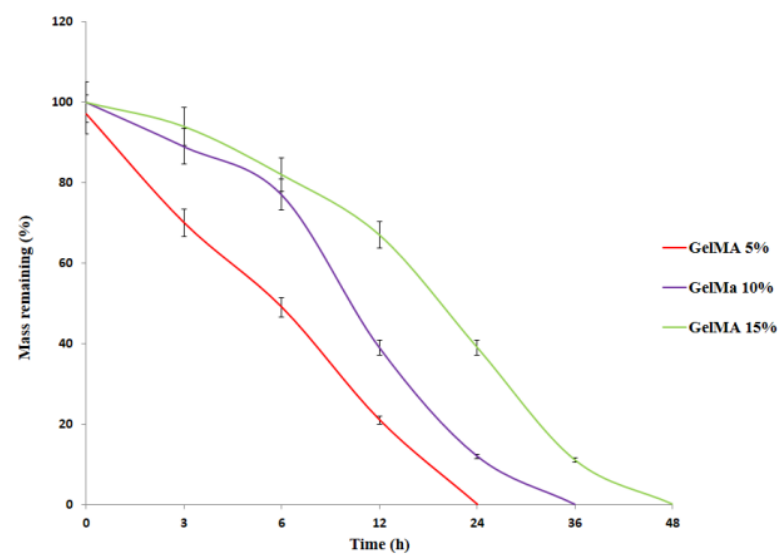

Figure 8. The degradation profile of GelMA at different concentrations.

\subsection{Degradation study.}

Hydrogels used as scaffolds for tissue engineering are chosen to degrade by body fluids after in vivo transplantation at a similar speed to one of targeted tissue formation. Hydrogel degradation could be induced by enzymes and chemicals [45]. After 24 hours of exposure to 
$2.5 \mathrm{U} / \mathrm{ml}$ collagenases at $37^{\circ} \mathrm{C}$, GelMA 5\% hydrogels were fully degraded. Conversely, more than half of the mass of GelMA $10 \%$ and GelMA $15 \%$ hydrogels remained after the enzymatic degradation test. We observed that the degradation rate decreased as the hydrogel concentration increased (Figure 8).

\section{Conclusions}

In this study, facile synthesis of GelMA was carried out using an aqueous medium. The preparation of GelMA in this way is cost-effective and easy to prepare in laboratory settings with high yield under optimal conditions. The characteristic functional group of aqueousmediated GelMA is correlated well with PBS and carbonate-bicarbonate-derived GelMA. Moreover, the physicochemical features of aqueous-mediated GelMA hydrogel governed by adjusting the gel concentration provide a tunable spectrum of degradation rate and swelling properties for downstream applications. Furthermore, it is established that the GelMA possesses cell growth supportive properties quite similar to gelatin and collagen. Thus, the aqueous-mediated GelMA hydrogels synthesized in this study may find many biomedical applications, particularly in tissue engineering as a scaffolding material, owing to their tunable physicochemical and other functional properties.

\section{Funding}

This research received no external funding

\section{Acknowledgments}

The authors gratefully acknowledge the VIT for providing the research and instrumentation facilities.

\section{Conflicts of Interest}

The authors declared no conflict of interest.

\section{References}

1. Han, F.; Wang, J.; Ding, L.; Hu, Y.; Li, W.; Yuan, Z.; Guo, Q.; Zhu, C.; Yu, L.; Wang, H.; Zhao, Z.; Jia, L.; Li, J.; Yu, Y.; Zhang, W.; Chu, G.; Chen, S.; Li, B. Tissue Engineering and Regenerative Medicine: Achievements, Future, and Sustainability in Asia. Frontiers in bioengineering and biotechnology 2020, 8, 83, https://doi.org/10.3389/fbioe.2020.00083.

2. Moysidou, C.-M.; Barberio, C.; Owens, R. M. Advances in Engineering Human Tissue Models. Frontiers in bioengineering and biotechnology. 2020, 620962, https://doi.org/10.3389/fbioe.2020.620962.

3. Zhu, L.; Luo, D.; Liu, Y. Effect of the Nano/Microscale Structure of Biomaterial Scaffolds on Bone Regeneration. International Journal of Oral Science 2020, 12, 6, https://doi.org/10.1038/s41368-020-0073y.

4. Hosseini, V.; Ahadian, S.; Ostrovidov, S.; Camci-Unal, G.; Chen, S.; Kaji, H.; Ramalingam, M.; Khademhosseini, A. Engineered Contractile Skeletal Muscle Tissue on a Microgrooved Methacrylated Gelatin Substrate. Tissue Engineering Part A 2012, 18, 2453-2465, https://doi.org/10.1089/ten.tea.2012.0181.

5. Goto, R.; Nishida, E.; Kobayashi, S.; Aino, M.; Ohno, T.; Iwamura, Y.; Kikuchi, T.; Hayashi, J.-I.; Yamamoto, G.; Asakura, M.; Mitani, A. Gelatin Methacryloyl-Riboflavin (GelMA-RF) Hydrogels for Bone Regeneration. International journal of molecular sciences 2021, 22, 1635, https://doi.org/10.3390/ijms22041635.

6. Sun, M.; Sun, X.; Wang, Z.; Guo, S.; Yu, G.; Yang, H. Synthesis and Properties of Gelatin Methacryloyl (GelMA) Hydrogels and Their Recent Applications in Load-Bearing Tissue. Polymers 2018, 10, https://doi.org/10.3390/polym10111290.

7. Hu, Q.; Tang, H.; Yao, Y.; Liu, S.; Zhang, H.; Ramalingam, M. Rapid Fabrication of Gelatin-Based Scaffolds 
with Prevascularized Channels for Organ Regeneration. Biomedical Materials 2021, 16, 45010, https://doi.org/10.1088/1748-605x/abef7b.

8. Madduma-Bandarage, U. S. K.; Madihally, S. V. Synthetic Hydrogels: Synthesis, Novel Trends, and Applications. Journal of Applied Polymer Science 2021, 138, 50376, https://doi.org/10.1002/app.50376

9. Rammal, H.; GhavamiNejad, A.; Erdem, A.; Mbeleck, R.; Nematollahi, M.; Emir Diltemiz, S.; Alem, H.; Darabi, M. A.; Ertas, Y. N.; Caterson, E. J.; Ashammakhi, N. Advances in Biomedical Applications of SelfHealing Hydrogels. Materials Chemistry Frontiers 2021, 5, 4368-4400, https://doi.org/10.1039/D0QM01099E.

10. Nicolas, J.; Magli, S.; Rabbachin, L.; Sampaolesi, S.; Nicotra, F.; Russo, L. 3D Extracellular Matrix Mimics: Fundamental Concepts and Role of Materials Chemistry to Influence Stem Cell Fate. Biomacromolecules 2020, 21, 1968-1994, https://doi.org/10.1021/acs.biomac.0c00045.

11. Krishnan, S.; Sekar, S.; Katheem, M. F.; Krishnakumar, S.; Sastry, T. Fish Scale Collagen-A Novel Material for Corneal Tissue Engineering. Artificial Organs 2013.

12. Cohen, N.; Toister, E.; Lati, Y.; Girshengorn, M.; Levin, L.; Silberstein, L.; Seliktar, D.; Epstein, E. Cell Encapsulation Utilizing PEG-Fibrinogen Hydrogel Supports Viability and Enhances Productivity under Stress Conditions. Cytotechnology 2018, 70, 1075-1083, https://doi.org/10.1007/s10616-018-0204-x.

13. Cañibano-Hernández, A.; Saenz Del Burgo, L.; Espona-Noguera, A.; Orive, G.; Hernández, R. M.; Ciriza, J.; Pedraz, J. L. Hyaluronic Acid Promotes Differentiation of Mesenchymal Stem Cells from Different Sources toward Pancreatic Progenitors within Three-Dimensional Alginate Matrixes. Molecular pharmaceutics 2019, 16, 834-845, https://doi.org/10.1021/acs.molpharmaceut.8b01126.

14. Montoya, C.; Du, Y.; Gianforcaro, A. L.; Orrego, S.; Yang, M.; Lelkes, P. I. On the Road to Smart Biomaterials for Bone Research: Definitions, Concepts, Advances, and Outlook. Bone Research 2021, 9, 12, https://doi.org/10.1038/s41413-020-00131-z.

15. Smoak, M. M.; Mikos, A. G. Advances in Biomaterials for Skeletal Muscle Engineering and Obstacles Still to Overcome. Materials Today Bio 2020, 7, 100069, https://doi.org/10.1016/j.mtbio.2020.100069.

16. Reddy, M. S.; Ponnamma, D.; Choudhary, R.; Sadasivuni, K. K. A Comparative Review of Natural and Synthetic Biopolymer Composite Scaffolds. Polymers 2021, https://doi.org/10.3390/polym13071105.

17. Klotz, B. J.; Gawlitta, D.; Rosenberg, A. J. W. P.; Malda, J.; Melchels, F. P. W. Gelatin-Methacryloyl Hydrogels: Towards Biofabrication-Based Tissue Repair. Trends in biotechnology 2016, 34, 394-407, https://doi.org/10.1016/j.tibtech.2016.01.002.

18. Tamayol, A.; Annabi, N.; Khademhosseini, A. Biomaterials Synthesis, Properties, and Biomedical Applications of Gelatin Methacryloyl (GelMA) Hydrogels. 2015, 73, 254-271, https://doi.org/10.1016/j.biomaterials.2015.08.045.

19. Xing, Q.; Yates, K.; Vogt, C.; Qian, Z.; Frost, M. C.; Zhao, F. Increasing Mechanical Strength of Gelatin Hydrogels by Divalent Metal Ion Removal. Scientific reports 2014, 4, 4706, https://doi.org/10.1038/srep04706.

20. Van Den Bulcke, A. I.; Bogdanov, B.; De Rooze, N.; Schacht, E. H.; Cornelissen, M.; Berghmans, H. Structural and Rheological Properties of Methacrylamide Modified Gelatin Hydrogels. Biomacromolecules 2000, 1, 31-38, https://doi.org/10.1021/bm990017d.

21. Nguyen, A. H.; McKinney, J.; Miller, T.; Bongiorno, T.; McDevitt, T. C. Gelatin Methacrylate Microspheres for Controlled Growth Factor Release. Acta biomaterialia 2015, 13, 101-110, https://doi.org/10.1016/j.actbio.2014.11.028.

22. Athirasala, A.; Lins, F.; Tahayeri, A.; Hinds, M.; Smith, A. J.; Sedgley, C.; Ferracane, J.; Bertassoni, L. E. A Novel Strategy to Engineer Pre-Vascularized Full-Length Dental Pulp-like Tissue Constructs. Scientific reports 2017, 7, 3323, https://doi.org/10.1038/s41598-017-02532-3.

23. Prakash Parthiban, S.; Rana, D.; Jabbari, E.; Benkirane-Jessel, N.; Ramalingam, M. Covalently Immobilized VEGF-Mimicking Peptide with Gelatin Methacrylate Enhances Microvascularization of Endothelial Cells. Acta Biomaterialia 2017, 51, 330-340, https://doi.org/10.1016/j.actbio.2017.01.046.

24. Kumar, H.; Sakthivel, K.; Mohamed, M.; Boras, E.; Shin, S.; Kim, K. Designing Gelatin Methacryloyl (GelMA)-Based Bioinks for Visible Light Stereolithographic 3D Biofabrication. Macromolecular Bioscience 2020, 21, https://doi.org/10.1002/mabi.202000317.

25. Abdollahi Baghban, S.; Ebrahimi, M.; Bagheri-Khoulenjani, S.; Khorasani, M. A Highly Efficient Microwave-Assisted Synthesis of an LED-Curable Methacrylated Gelatin for Bio Applications. RSC Advances 2021, 11, 14996-15009, https://doi.org/10.1039/D1RA01269J.

26. Zhu, M.; Wang, Y.; Ferracci, G.; Zheng, J.; Cho, N.-J.; Lee, B. H. Gelatin Methacryloyl and Its Hydrogels with an Exceptional Degree of Controllability and Batch-to-Batch Consistency. Scientific Reports 2019, 9, 686, https://doi.org/10.1038/s41598-019-42186-x.

27. Shirahama, H.; Lee, B. H.; Tan, L. P.; Cho, N.-J. Precise Tuning of Facile One-Pot Gelatin Methacryloyl (GelMA) Synthesis. Scientific Reports 2016, 6, 31036, https://doi.org/10.1038/srep31036.

28. Shahidi, S.; Janmaleki, M.; Riaz, S.; Sanati Nezhad, A.; Syed, N. A Tuned Gelatin Methacryloyl (GelMA) Hydrogel Facilitates Myelination of Dorsal Root Ganglia Neurons in Vitro. Materials Science and Engineering: C 2021, 126, 112131, https://doi.org/10.1016/j.msec.2021.112131.

29. Claaßen, C.; Claaßen, M. H.; Truffault, V.; Sewald, L.; Tovar, G. E. M.; Borchers, K.; Southan, A. 
Quantification of Substitution of Gelatin Methacryloyl: Best Practice and Current Pitfalls. Biomacromolecules 2018, 19, 42-52, https://doi.org/10.1021/acs.biomac.7b01221.

30. Zhou, L.; Tan, G.; Tan, Y.; Wang, H.; Liao, J.; Ning, C. Biomimetic Mineralization of Anionic Gelatin Hydrogels: Effect of Degree of Methacrylation. RSC Adv. 2014, 4, 21997-22008, https://doi.org/10.1039/C4RA02271H.

31. Hashim, D. M.; Man, Y. B. C.; Norakasha, R.; Shuhaimi, M.; Salmah, Y.; Syahariza, Z. A. Potential Use of Fourier Transform Infrared Spectroscopy for Differentiation of Bovine and Porcine Gelatins. Food Chemistry 2010, 118, 856-860, https://doi.org/10.1016/j.foodchem.2009.05.049.

32. Leu Alexa, R.; Iovu, H.; Ghitman, J.; Serafim, A.; Stavarache, C.; Marin, M.-M.; Ianchis, R. 3D-Printed Gelatin Methacryloyl-Based Scaffolds with Potential Application in Tissue Engineering. Polymers 2021, 13, 727, https://doi.org/10.3390/polym13050727. .

33. Krishnamoorthy, S.; Noorani, B.; Xu, C. Effects of Encapsulated Cells on the Physical-Mechanical Properties and Microstructure of Gelatin Methacrylate Hydrogels. International journal of molecular sciences 2019, 20, https://doi.org/10.3390/ijms20205061.

34. Hjortnaes, J.; Camci-Unal, G.; Hutcheson, J. D.; Jung, S. M.; Schoen, F. J.; Kluin, J.; Aikawa, E.; Khademhosseini, A. Directing Valvular Interstitial Cell Myofibroblast-Like Differentiation in a Hybrid Hydrogel Platform. Advanced healthcare materials 2014, 1-10, https://doi.org/10.1002/adhm.201400029.

35. Hu, X.; Ma, L.; Wang, C.; Gao, C. Gelatin Hydrogel Prepared by Photo-Initiated Polymerization and Loaded with TGF- $\beta 1$ for Cartilage Tissue Engineering. Macromolecular Bioscience 2009, 9, 1194-1201, https://doi.org/10.1002/mabi.200900275.

36. Nichol, J. W.; Koshy, S. T.; Bae, H.; Hwang, C. M.; Yamanlar, S.; Khademhosseini, A. Cell-Laden Microengineered Gelatin Methacrylate Hydrogels. Biomaterials 2010, 31, 5536-5544, https://doi.org/10.1016/j.biomaterials.2010.03.064.

37. Aldana, A. A.; Malatto, L.; Rehman, M. A. U.; Boccaccini, A. R.; Abraham, G. A. Fabrication of Gelatin Methacrylate (GelMA) Scaffolds with Nano- and Micro-Topographical and Morphological Features. Nanomaterials (Basel, Switzerland) 2019, 9, https://doi.org/10.3390/nano9010120.

38. Modaresifar, K.; Hadjizadeh, A.; Niknejad, H. Design and Fabrication of GelMA/Chitosan Nanoparticles Composite Hydrogel for Angiogenic Growth Factor Delivery. Artificial Cells, Nanomedicine, and Biotechnology 2018, 46, 1799-1808, https://doi.org/10.1080/21691401.2017.1392970.

39. Nireesha, G. R.; Divya, L.; Sowmya, C.; Venkateshan, N.; Babu, M.; Vuppalapati, L. Lyophilization/Freeze Drying-An Review. 2013, 3, 87-98.

40. Sornkamnerd, S.; Okajima, M. K.; Kaneko, T. Tough and Porous Hydrogels Prepared by Simple Lyophilization of LC Gels. ACS Omega 2017, 2, 5304-5314, https://doi.org/10.1021/acsomega.7b00602.

41. Tiwari, A.; Ramalingam, M.; Kobayashi, H.; Turner, A. Biomedical Materials and Diagnostic Devices. 2012, https://doi.org/10.1002/9781118523025.

42. Ostrovidov, S.; Annabi, N.; Seidi, A.; Ramalingam, M.; Dehghani, F.; Kaji, H.; Khademhosseini, A. Controlled Release of Drugs from Gradient Hydrogels for High-Throughput Analysis of Cell-Drug Interactions. Analytical Chemistry 2012, 84, 1302-1309, https://doi.org/10.1021/ac202256c.

43. Ramalingam, M.; Jabbari, E.; Ramakrishna, S.; Khademhosseini, A. Micro and Nanotechnologies in Engineering Stem Cells and Tissues 2013, https://doi.org/10.1002/9781118574775.

44. Sivashankar, S.; Young, A. T.; Erb, P. D.; Ligler, F. S.; Daniele, M. A. Characterizing the Swelling of Gelatin Methacrylamide and Effects on Microscale Tissue Scaffold Fabrication. In 2017 IEEE 12th International Conference on Nano/Micro Engineered and Molecular Systems (NEMS), 2017, 554-557, https://doi.org/10.1109/NEMS.2017.8017083.

45. Xiao, L.; Lin, J.; Chen, R.; Huang, Y.; Liu, Y.; Bai, J.; Ge, G.; Shi, X.; Chen, Y.; Shi, J.; Aiqing, L.; Yang, H.; Geng, D.; Wang, Z. Sustained Release of Melatonin from GelMA Liposomes Reduced Osteoblast Apoptosis and Improved Implant Osseointegration in Osteoporosis. Oxidative Medicine and Cellular Longevity 2020, 2020, 6797154, https://doi.org/10.1155/2020/6797154. 